\title{
Formação profissional em saúde: abordagem curricular de um curso de fisioterapia
}

\section{Vocational training in health: curricular approach to a physiotherapy course}

\author{
${ }^{1}$ Josiane Moreira Germano j_mg87@yahoo.com.br \\ 2 Patrícia Fernanda Sandrini \\ ${ }^{3}$ Adriana Regina de Jesus Santos
}

\section{RESUMO}

A mudança do modelo de saúde somado à alteração do perfil epidemiológico no Brasil fez com que a formação profissional fosse alvo de estudos devido à insuficiência do padrão de formação no acolhimento das demandas da saúde pública. Desta forma, os objetivos deste artigo são: discorrer sobre a formação profissional em Fisioterapia na perspectiva do paradigma sanitário e apresentar a análise do Projeto Político Pedagógico de um curso de Fisioterapia e suas repercussões na formação profissional de fisioterapeutas egressos. Trata-se de um estudo de análise documental de caráter qualitativo, com levantamento bibliográfico de artigos em bases de dados como: Scielo e Lilacs, além de outros manuscritos, sem restrição de ano buscou-se embasamento para a construção do texto, artigos relacionados à formação profissional em saúde, currículo em fisioterapia e diretrizes curriculares nacionais em saúde. Com esta pesquisa foi possível perceber que a fisioterapia nasce de uma perspectiva exclusivamente reabilitadora onde o foco de formação está voltado ao cuidado hospitalar e clínico. A análise do Projeto Político Pedagógico possibilita perceber que apesar de três readequações curriculares dos eixos de conhecimento, o curso apresenta características majoritariamente biológicas e instrumentais. O currículo segue uma vertente tradicionalista onde as disciplinas são organizadas de modo linear e por séries, dificultando a integração disciplinar. Para contemplar a necessidade do modelo de saúde vigente percebe-se a necessidade de direcionar a formação para aspectos mais humanos, onde o curso tem o desafio de reorientar o currículo aproximando-o das necessidades reais do paradigma sanitário.

Palavras chave: Formação Profissional. Currículo. Fisioterapia.

\begin{abstract}
The change of the health model added to the alteration of the epidemiological profile in Brazil caused that the professional formation was the object of studies due to the insufficiency of the training pattern in the reception of the demands of the public health. Thus, the objectives of this article are: to discuss the professional training in Physical Therapy from the perspective of the health paradigm and to present the analysis of the Political Pedagogical Project of a Physical Therapy course and its repercussions in the professional training of physiotherapists. It is a qualitative documentary analysis study, with a bibliographical survey of articles in databases such as: Scielo and Lilacs, in addition to other manuscripts, without restraint of the year, we sought to build the text, articles related to Professional training in health, curriculum in physical therapy and national curricular guidelines in health. With this research it was possible to perceive that physiotherapy arises from an exclusively rehabilitating perspective where the focus of training is focused on

1 Fisioterapeuta Especialista em Saúde da Família pela Faculdade de Medicina em Marília.

2 Fisioterapeuta Especialista em Fisioterapia Neonatal e Pediátrica, pela Faculdade Inspirar, Curitiba.

3 Pós Doutora em Educação pela Universidade Federal Fluminense. Docente dos Cursos de Graduação e Strictu Sensu em Educação da Universidade Estadual de Londrina.
\end{abstract}


hospital and clinical care. The analysis of the Pedagogical Political Project makes it possible to perceive that despite three curricular adjustments of the axes of knowledge, the course presents mostly biological and instrumental characteristics. The curriculum follows a traditionalist strand where the disciplines are organized in a linear and series way, making disciplinary integration difficult. In order to contemplate the need for the current health model, it is necessary to direct the training to more human aspects, where the course has the challenge of reorienting the curriculum, bringing it closer to the real needs of the health paradigma.

Keywords: Vocational Training. Curriculum. Physiotherapy.

\section{INTRODUÇÃO}

O modelo com que os serviços de saúde se organizam atrelados ao modelo econômico e político tem influenciado diretamente a formação dos profissionais, sobretudo da saúde (CATANI; OLIVEIRA; DOURADO, 2001). Posto isso, o papel a ser desenvolvido pelos trabalhadores podem variar entre o atendimento às exigências do mercado e o protagonismo na reversão da realidade epidemiológica.

Somado a incidência de portadores de sequelas de poliomielite, pessoas com distúrbios motores e o aumento dos acidentes de trabalho, a Fisioterapia emergiu no Brasil a partir de 1929, com a criação do primeiro curso técnico em São Paulo, sendo regulamentada apenas em 1969, em meio à ditadura militar (PEREIRA; ALMEIDA, 2006). Neste período, a compreensão de saúde era vista como instrumento de sustentação econômica e não como direito social da população (BISPO JUNIOR 2009).

Conforme Moreira e Silva (1997, p. 28), “o currículo é um terreno de produção e de política cultural, no qual os materiais existentes funcionam como matéria prima de criação e recriação e, sobretudo, de contestação e transgressão". O currículo escolar tem ação direta ou indireta na formação e desenvolvimento do discente. Assim, é fácil perceber que a ideologia, cultura e poder nele configurados são determinantes no resultado educacional que se produzirá.

Desta forma, justifica-se e relevância de estudos que abordem a formação profissional e currículo, pois com a construção do Sistema Único de Saúde (SUS), fez-se necessário (re)pensar a concepção de formação profissional e organizações curriculares tradicionalmente alicerçados na perspectiva biologicista de cuidado reduzindo o indivíduo à doença.

Essa visão de saúde é vista como ausência de doença, e influenciou o aprendizado centrado no hospital, com caráter médico-hegemônico, privado e especialista, reduzindo a oportunidade de outros espaços e perspectivas de aprendizagem, como a atuação a Atenção Primária à Saúde, por exemplo. Portanto as questões a serem problematizadas neste artigo serão: O panorama da formação em Fisioterapia e como é a organização e perspectiva de currículo de um curso de graduação em Fisioterapia no Paraná?

Com intuito de responder a questão problema, os objetivos deste artigo são: discorrer sobre a formação profissional em Fisioterapia na perspectiva do paradigma sanitário e apresentar a análise de Projeto Político e Pedagógico (PPP) de um curso de Fisioterapia no Paraná, e suas repercussões na formação profissional de fisioterapeutas egressos. Para tanto, este artigo inicialmente trouxe uma breve análise do panorama e influências na formação profissional em saúde sob a ótica do modelo capitalista.

Posteriormente, foi relatado brevemente sobre o início da Fisioterapia no Brasil, com e a formação em Fisioterapia, aumento das Universidades e dos cursos ofertados inicialmente, em instituições públicas e depois, o aumento dos cursos de Fisioterapia por meio da privatização da Educação Superior. 
Em outro momento, destaca-se o modelo de formação e perfil profissional, cuja função inicial centrava-se na reabilitação e as necessidades de mudança na formação. Fato que pode ser destacado em 2002, onde o Conselho Nacional de Educação instituiu as Diretrizes Curriculares Nacionais do curso de graduação em Fisioterapia. E por fim, destaca-se o que é currículo, suas formas de organização, e uma análise do currículo de um curso de Fisioterapia no Paraná.

\section{PROCEDIMENTOS METODOLÓGICOS}

O caminho metodológico está pautado nos princípios da pesquisa qualitativa, descritos por Bogdan e Biklen (1982) e citados por Lüdke e André (1986), que abordam algumas características básicas como: os dados predominantemente descritivos, onde a preocupação com o processo é muito maior do que com o produto e a análise dos dados tende a seguir um processo indutivo (BOGDAN; BIKLEN, 1982).

O conjunto de procedimentos de apoio à investigação foi formado por levantamento bibliográfico que permite o aprofundamento e ampliação dos pressupostos teóricos (LAKATOS; MARCONI, 1987; CERVO; BERVIAN, 1976). Realizou-se análise documental, onde se considera, entre outros fatores, o contexto político e sócio-cultural, no qual se construíram os valores do documento, os interesses confessos ou velados, para quem e por quem foi escrito (SUMIYA, 2014).

O documento analisado foi o PPP de um curso de Fisioterapia do Norte do Paraná, o que possibilitou a reflexão deste documento sobre o contexto do currículo construído no espaço da formação profissional. A análise documental permitiu situar a instituição de ensino no seu processo pedagógico e educativo, possibilitando, assim, entender a relação do currículo vivido com o currículo oficial, bem como suas implicações no contexto de formação de acordo com o paradigma sanitário.

\section{PANORAMA E INFLUÊNCIAS NA FORMAÇÃO PROFISSIONAL EM SAÚDE}

Para discorrer sobre a formação do fisioterapeuta faz-se necessário entender o contexto que está alicerçada a formação em saúde, no entanto, é necessário voltar ao final do século XIX, período que trouxe inúmeros avanços tecnológicos no setor saúde, como por exemplo, as descobertas de Koch e Pasteur no campo da microbiologia substituindo o empirismo pelo método científico (AGUIAR, 2003).

A rápida proliferação das práticas assistenciais suscitaram reflexões de um processo de contenção regulamentada. Abraham Flexner, médico americano, surge neste contexto e assume, em 1910 onde publicou o Relatório Flexner que influenciou não só o ensino norte-americano como as práticas médicas mundiais, consolidando o paradigma da medicina científica, que se faz presente na formação dos profissionais de saúde. (GONZÁLEZ; ALMEIDA, 2010).

A formação médica e o modelo de assistência ajustados sob essa ótica revelava diversas influências, como o mecanicismo, o biologismo, o individualismo, a especialização e o curativismo (AGUIAR, 2003). De acordo com Pagliosa e Da Ros (2008) as contribuições do modelo flexneriano são consideradas importantes para a educação médica, porém a ênfase dada ao modelo curativista alicerçado na doença e no hospital conduziu os programas educacionais a uma visão reducionista do ser humano.

Segundo Tancredi e Feuerwerker (2001) o modelo positivista flexneriano começou a ser implantado no Brasil na década de 40 e influenciou os cursos de medicina, odontologia e enfermagem e, além de tudo, reformulou os cursos já existentes. Centrado no hospital, com gastos exorbitantes, consumo desenfreado das possibilidades de diagnóstico e tratamento, este modelo mostrou a incapacidade de alterar os atos do cotidiano os 
quais determina o modo da produção da saúde e colocou em cheque a lógica do procedimento médico centrado (MEHRY; MALTA; SANTOS, 2004; SAKAI, 2001).

Destarte, é possível perceber que tradicionalmente as profissões da saúde têm em seu cerne a formação calcada no modelo neoliberal capitalista (PAIM, 2003). Segundo Paim (2002) a formação com essa perspectiva fez com que aumentasse o gargalo da produção de empregos no setor terciário cujo enfoque é incompatível com a realidade e demandas de saúde da maior parte da população.

Em função do modelo flexneriano que reduz o indivíduo a um organismo biológico, questiona-se o preparo dos profissionais da saúde para o exercício das suas funções de modo a contemplar os preceitos do SUS. Dessa forma, vem se configurando uma nova abordagem, um novo paradigma chamado paradigma da integralidade, biopsicossocial ou paradigma sanitário (ARAÚJO, 2005).

O SUS, criado na década de 1980, encerra a lógica de atuação fragmentada e entende que o modelo sanitário mostra que há necessidade de que o aprendizado extrapole as habilidades técnico-instrumentais e avance em direção das capacidades relacionais e sociais (MARCO, 2006). Desta forma para Da Ros (2000), (re) pensar a formação de recursos humanos para a saúde é necessidade prioritária, porém sempre foi relegado ao segundo plano nos processos de reforma, as questões de educação ficaram à margem da reorganização dos serviços e da redefinição das práticas de atenção.

Para que o novo paradigma sanitário se efetive necessita-se investir na readequação da formação, por meio da reestruturação dos currículos e reaproximar as metodologias de ensino para promover uma formação crítica e reflexiva inclusive para os profissionais que já atuam nos serviços (PEREIRA; BARROS; AUGUSTO, 2011).

Discorrido sobre a formação em saúde e entendido o contexto de sua concepção, a formação e a prática do fisioterapeuta também foram influenciadas pelo modelo flexneriano que direcionou prioritariamente o foco de atenção para clínicas de reabilitação e complexos hospitalares. Tradicionalmente, é notória a tendência no Ensino Superior em Fisioterapia a valorização do individual, da terapêutica, da especialidade e a utilização de métodos e técnicas sofisticadas (REZENDE, 2007).

Tais características estão em consonância com o modelo médico-assistencial privatista, ainda hegemônico no Brasil. A forma de perceber, explicar e enfrentar os problemas de saúde ao longo da história do nosso país acarretou o predomínio do pensamento clínico sobre o processo saúde-doença e, consequentemente, os esforços dos profissionais ou áreas de estudo preocupadas com as condições de saúde do homem concentraram-se, por um extenso período, na descoberta de novos métodos de “tratamento" das doenças, revelando a preponderância de uma assistência “curativa”, recuperativa e reabilitadora (RODRIGUES, 2008, p.104).

É sabido que o projeto neoliberal promoveu a iniciativa privada, diversos cursos da área de saúde, sobretudo, os cursos de Fisioterapia expandiram-se sem planejamento e regulação, em meio à estagnação do setor público quando comparado às instituições privadas (BISPO JUNIOR, 2009; PEREIRA; ALMEIDA, 2006).

Em relação à natureza administrativa das instituições de ensino, verifica-se que a expansão da Fisioterapia ocorreu majoritariamente a partir da iniciativa privada. Em 1995, dos 63 cursos existentes, 46 (73\%) eram da rede privada e 17 (27\%), da rede pública. Em 2003, esses números eram 263 (88,2\%) e 35 (11,8\%), respectivamente, demonstrando a tendência à privatização do ensino da Fisioterapia (PEREIRA; ALMEIDA, 2006). Embora se deva destacar que a rede pública ampliou o número de cursos em mais de 100\% no período observado, o aumento se apresenta de forma muito mais expressiva na rede privada (BISPO JUNIOR, 2009).

Os dados apontam que o processo de privatização dos cursos do ensino superior direcionam a formação em consonância com o modelo hegemônico, porém, para que a atuação seja capaz de contemplar o paradigma 
sanitário, a Fisioterapia precisa aproximar-se do campo da Promoção da Saúde e do movimento da Saúde Coletiva, conciliando com as práticas de reabilitação. Para Bispo Junior (2009, p.5) "esse redimensionamento do objeto de intervenção e da práxis profissional conduz a mudanças mais profundas, de natureza epistemológica, na concepção e atuação do fisioterapeuta".

\section{CURRÍCULO}

O currículo é um modelo abstrato que define as experiências e resultados de uma escola ou de um sistema escolar. Este modelo ganha forma em documento, mas, em termos mais vastos, existe nas percepções partilhadas dos que participam na instrução e respectivas comunidades. Este modelo define tanto uma arena na qual as tensões entre a escola e sociedade são resolvidas como um conjunto de normas para controlar o ensino (PACHECO, 2005).

Desde suas origens, o currículo se comporta como um instrumento que tem a capacidade de estruturar a escolarização, a vida nos centros educacionais e as práticas pedagógicas por transmitir regras e normas determinantes (SACRISTÁN, 2013). Como em qualquer campo do conhecimento, nos estudos curriculares existem diferentes classificações de teorias curriculares, que não são mais do que tentativas de abordagens das concepções de currículo por meio das quais se diferenciam formas de relacionar a teoria com a prática e a escola com a sociedade (SANTOS, 2008).

A construção curricular no Brasil não se deu sob uma única ideologia, mas com influência de tendências, objetivos e interesses diferentes. Segundo McNeil (2001) o currículo pode ser classificado em quatro abordagens distintas: Acadêmico, Humanista, Tecnológico e Reconstrucionista, que foram sendo construídas ao longo do tempo (SANTOS, 2008).

Perante as variedades de propostas de conceptualização da realidade curricular, Kemmis (1988), formula as teorias técnica, prática e crítica, as raízes dessa proposta encontram-se na teoria dos interesses construtivos de saberes de Habermas, aplicada à educação, dentre outros, por Grundy, Apple e Macdonald. Porém deve reconhecer-se que Macdonald, foi o primeiro a propor uma teorização curricular, na base dos interesses técnico, prático, crítico, identificando a teoria de controlo, consenso e emancipação - para justificar a proposta que faz relativamente aos modelos de desenvolvimento curricular: linear, consensual e dialógico (PACHECO, 2005).

\section{CURRÍCULO E PROPOSTA DE FORMAÇÃO PROFISSIONAL EM FISIOTERAPIA DE UMA UNIVERSIDADE ESTADUAL NO PARANÁ}

A discussão sobre a regulação da formação dos profissionais de saúde perdura desde 1930 em diversos momentos no Brasil (PEREIRA, 2013). De acordo com Rezende (2013) o movimento ganhou corpo na década de 1990, com a chegada das DCNs que entendeu a urgência de ligar a formação Às necessidades sociais em consonância aos princípios do SUS. Para as profissões de saúde, as DCNs remeteram a necessidade de mudança de rumo no que se refere à formação profissional, segundo Ceccim e Carvalho (2006, p.88) “constata-se a necessidade de se voltar para os sentidos, os valores e os significados do que se faz e para quem se dirige nossa ação".

Para discutir os sentidos no novo paradigma, tomaram-se como referência as diretrizes dos cursos de Enfermagem, Medicina e Odontologia, justifica-se a escolha desses cursos pelos seguintes critérios: os três cursos contemplam a base da Equipe de Referência da Estratégia de Saúde da Família ${ }^{4}$ (MOREIRA; DIAS, 2015).

4 Estratégia criada pelo Ministério da Saúde em 1994 para promover a reorganização das ações de atenção primária no país (AGUIAR, 2007). 
O objetivo de formar um profissional nesta perspectiva generalista, crítica e reflexiva, com vistas a integralidade corrobora com o conceito ampliado de saúde culminando em outra visão do próprio SUS, ao incluir as ações de promoção, prevenção, responsabilidade social e compromisso com a cidadania, fica claro que o foco da formação desloca-se do cunho absolutamente curativo (ROSSINI; LAMPERT, 2004; MOREIRA; DIAS, 2015).

Nas DCNs do curso de Enfermagem, atenta-se que "o estímulo às dinâmicas de trabalho em grupos, por favorecerem a discussão coletiva e as relações interpessoais” (BRASIL, 2001a). Posto isso, entende-se que é necessário outro olhar para as práticas metodológicas de ensino-aprendizagem como Aprendizagem Baseada em Problemas, por exemplo (MOREIRA; DIAS, 2015).

Em relação a Fisioterapia, o Conselho Nacional de Educação instituiu, em 2002, as Diretrizes Curriculares Nacionais do Curso de Graduação em Fisioterapia, marco importante na orientação e transformação do ensino em que são definidos princípios, fundamentos e condições para a formação em todas as instituições nacionais de ensino (BRASIL, 2003). O perfil do profissional egresso deve revelar-se generalista com formação crítica, humanista e reflexiva e destaca que a responsabilidade da atenção à saúde não se encerra com o ato técnico, mas sim com a resolução do problema em âmbito individual e coletivo (BRASIL, 2003).

A análise do $\mathrm{PPP}^{5}$ do curso pioneiro em Fisioterapia implantado no Paraná, no final da década de 1970, cuja formação do Departamento do curso, que fora administrada pela Medicica, aconteceu em 1983 com a presença de aproximadamente quinze docentes naquela época.

O currículo deste curso sofreu três mudanças, a primeira foi o aumento para quatro anos letivos onde as disciplinas foram (re) divididas em ciclos básico, clínico e profissionalizante, a segunda como consequência, foi o aumento da carga horária, que saltou de 2.655 horas para 4.038h, permanecendo sem mudanças até 1992 . A terceira mudança aconteceu em 2006, onde os conhecimentos foram divididos em quatro eixos: Ciências Sociais e Humanas (7,5\% do total do curso); Conhecimentos Biotecnológicos (5,3 \% do total do curso); Ciências Biológicas e da Saúde (18,7 \% do total do curso); e Conhecimentos fisioterápicos (68,5 \% do total do curso) e ainda, houve um novo aumento da carga horária, sendo 5\% destinada às atividades complementares.

Como foi descrito anteriormente, alicerçar a formação ao encontro do modelo vigente em saúde é um dos caminhos para a reafirmação do SUS, portanto a disciplina de Saúde Coletiva tem a responsabilidade de integrar seus conhecimentos como principais elos de mudança curricular, destarte, observou-se que disciplina de Saúde Coletiva I, II e III do currículo de 2006, aborda os seguintes assuntos: (I) Concepção do processo saúde e doença; Políticas de Saúde Internacional, Nacional e Local; Epidemiologia e Atuação da Fisioterapia na Atenção Integral à Saúde, (II) vivências na UBS para análises das condições de saúde da população adstrita e ainda, afirmar o papel do fisioterapeuta neste serviço de saúde. Enquanto a disciplina Saúde Coletiva III com o tema central: Domicílio e Família; Análise das condições de saúde e o papel do fisioterapeuta no serviço de saúde. Por fim, na quarta série, a Disciplina Saúde Coletiva IV é colocado em prática o desenvolvimento de atividades de aprendizagem profissional em situações reais de ação do fisioterapeuta em saúde coletiva cuja carga horária abarca 170 horas.

Desta forma, ao discorrer sobre este currículo é possível perceber que a disciplina de Saúde Coletiva é contemplada no eixo de conhecimento: Ciências sociais e humanas por proporcionar ao discente o estudo do homem e de suas relações sociais; a compreensão do processo saúde doença, abarcando a integração dos aspectos psicossociais, culturais, filosóficos, antropológicos e epidemiológicos, norteados pelos princípios éticos. Portanto observa-se que este eixo apresenta a menor quantidade de carga horária disponível quando comparado aos outros eixos, reforçando a lacuna da inserção dos aspectos sociais da saúde na formação, onde prioriza-se o enfoque curativo em sua composição (UTIDA et al, 2012).

5 * Projeto Político Pedagógico do curso de Fisioterapia, ano: 2014. Disponível em: http://www.uel.br/ ccs/fisioterapia/ 
Contudo, o currículo observado prevê a duração mínima e máxima prevista para o curso de Fisioterapia para 4 (quatro) e 8 (oito) anos, respectivamente, corroborando com os requisitos das DCNs. Para obter o grau de Fisioterapeuta, o estudante deverá cumprir um total de 4.060 (quatro mil e sessenta) horas relativas ao currículo pleno proposto incluindo as 100 (cem) horas destinadas ao cumprimento de Atividades Acadêmicas Complementares.

O currículo analisado corrobora com os dados encontrados na pesquisa de Utida e colaboradores (2012) que apontam que a estrutura curricular está pautada em disciplinas compartimentadas com pouca integração entre as mesmas, contrariando a ideia de interdisciplinaridade, perpetuando a vertente tradicionalista engessando a comunicação disciplinar (SUMIYA, 2014).

De acordo com Santomé (1998), a configuração linear disciplinar é criticada como currículo quebra-cabeças. Desta maneira, o conhecimento se operacionaliza por fragmentos, dentro de uma estrutura rígida levando os estudantes a enxergar determinada disciplina como um conhecimento autônomo com dificuldade de levá-los para a realidade (SANTOMÉ, 1998).

O currículo estudado apresentou três mudanças em suas diretrizes curriculares, porém, na perspectiva de Bispo Junior (2009), o modelo de formação mantém a ótica curativa e reabilitadora de cunho privatista, fomentando a formação de fisioterapeutas a partir da concepção de profissional liberal. O estimulo ao desenvolvimento profissional na visão de Schwingel (2002) é tecnicista e foi alicerçado ao discurso da crescente concorrência do mercado de trabalho que reforçou o domínio do conhecimento biológico e das técnicas de reabilitação como exigência suficiente e necessária para instalação no mercado de trabalho.

Diante deste contexto, na década de 1990 a Fisioterapia progrediu em relação ao status profissional e ampliou suas especializações e seu campo de atuação (BISPO JUNIOR, 2009). Direcionou sua atuação para o sistema suplementar, convênios e planos de saúde e ainda, aperfeiçoou suas especialidades como: Dermatofuncional, Reeducação Postural Global, Pilates e Acupuntura, crivando a sua atuação para uma população mais seleta (SILVA KAIK; SILVA SOUSA, 2010; BISPO JUNIOR, 2009).

A valorização do nível terciário de atenção contribuiu para a dificuldade de ampliação do campo de atuação da Fisioterapia na Atenção Básica, em virtude do inadequado perfil profissional para atuação nesta perspectiva (BISPO JUNIOR, 2009). Silva e Da Ros (2007) apontam a limitada compreensão, dos profissionais e estudantes, das possibilidades de atuação do fisioterapeuta na Atenção Básica à Saúde.

De acordo com Meyer, Costa e Gico (2006) a organização curricular e o método de ensino dos cursos de Fisioterapia não estão alinhados com as demandas sociais e com as políticas públicas de saúde, os autores citam que há pouco envolvimento dos discentes e docentes em Fisioterapia com as políticas institucionais e governamentais, porém, no início do século XXI houveram algumas iniciativas, como a criação da Associação Brasileira de Ensino em Fisioterapia (ABENFISIO) que suscitaram necessidade de (re) pensar do modelo de formação e perfil profissional do fisioterapeuta que congregam discentes e docentes que se dedicam em debater e construir a formação em Fisioterapia sobre outra ótica, para isso, semestralmente organiza fóruns nacionais a respeito do ensino, nos quais se realizam avaliações e discutem a qualidade e o modelo de formação com vistas a uma formação fundamentada nos princípios do SUS e articulada com os saberes da Saúde Coletiva (BISPO JUNIOR, 2009).

\section{CONSIDERAÇÕES FINAIS}

Diante da discussão posta, é possível observar que a formação em Fisioterapia, segue a lógica flexneriana, que visa um modelo de currículo voltado a práticas h. Este modelo de currículo influenciou todas as outras profissões hegemônicas de cunho biologicista. Em meio à perspectiva capitalista o curso de fisioterapia aproxi- 
mou sua formação do setor terciário supervalorizando as especialidades como: Acupuntura, Dermatofuncional e Reeducação Postural Global direcionando suas práticas para o mercado privado.

Com o neoliberalismo fortemente marcado na década de 1990 é possível observar o aumento dos cursos de Fisioterapia, sobretudo do setor privado, alicerçando a formação no caráter reabilitador e afastando os currículos da formação integrada, social e humana. Contudo, as Diretrizes Curriculares em 2002, despertam que a formação deve atender os preceitos do SUS, ou seja, olhar para o indivíduo dentro de seu contexto social.

Apesar dos avanços, percebe-se a necessidade de direcionar a formação para aspectos mais humanos, onde o curso tem o desafio de reorientar o currículo aproximando-o das necessidades reais do paradigma sanitário.

\section{REFERÊNCIAS}

AGUIAR, Raphael Augusto Teixeira de. A construção internacional do conceito de atenção primária à saúde (APS) e sua influência na emergência e consolidação do sistema único de saúde no Brasil. 136f. Dissertação (Mestrado em Saúde Pública) - Universidade Federal de Minas Gerais, Belo Horizonte, 2003. Disponível em: http://hdl.handle.net/1843/ECJS-76RHW7. Acesso em: 10 jan.2017.

ARAÚJO, Dolores Gonçalves Borges. Contribuição para a humanização da educação médica: o diário de campo como instrumento de avaliação qualitativa. Dissertação (Mestrado em Medicina e Saúde). Universidade Federal da Bahia, Salvador, 2005.

BISPO JUNIOR, José Patrício. Formação em fisioterapia no Brasil: reflexões sobre a expansão do ensino e os modelos de formação. História Ciência e Saúde - Manguinhos, vol.16, n.3, p.655- 668, 2009. Disponível em: http://dx.doi.org/10.1590/S0104-59702009000300005 . Acesso em: 10 jan. 2017.

BOGDAN, Robert; BIKLEN, Sari Knopp. Qualitative research for education. In: LÜDKE, Menga; ANDRÉ, Marli. Pesquisa em Educação: abordagens qualitativas. São Paulo: EPU, 1982.

BRASIL. Conselho Nacional de Educação. Parecer CNE/CES n 8/2007. Dispõe sobre carga horária mínima e procedimentos relativos à integralização e duração dos cursos de graduação, bacharelados, na modalidade presencial. Diário Oficial da União. 2007. Disponível em: http://portal.mec.gov.br/cne. Acesso em: 20 jan. 2017.

BRASIL. Ministério da Saúde. A educação permanente entra na roda: polos de educação permanente em saúde: conceitos e caminhos a percorrer. 2 ed. Brasília: Ministério da Saúde, 2005. Disponível em: http:// bvsms.saude.gov.br/bvs/publicacoes/educacao_permanente_entra na_roda.pdf. Acesso em: 10 jan, 2017.

BRASIL. Conselho Nacional de Educação. Diretrizes curriculares nacionais do curso de graduação em fisioterapia. In: Almeida, Márcio. Diretrizes curriculares nacionais para os cursos universitários da área de saúde. Londrina: Rede Unida. p.30-36, 2003.

BRASIL. Conselho Federal de Educação. Resolução 4. Parecer CNE/CES 583. Fixa os mínimos de conteúdos e duração dos cursos de fisioterapia e terapia ocupacional. Diário Oficial da União, Brasília, 4 de abril de 2001. Disponível em: http://portal.mec.gov.br/cne/arquivos/pdf/CES0108.pdf. Acesso em: 20 jan. 2017.

BRASIL. Conselho Federal de Educação. Resolução 4. Fixa os mínimos de conteúdos e duração dos cursos de fisioterapia e terapia ocupacional, 1983. Disponível em: http://www.prolei.inep.gov.br/pesquisar. do;jsessionid= A05389F814EE4C24BA 603F755C800E7D?codThesaurus=37179. Acesso em: 10 jan.2017. 
BRASIL. Decreto-lei 938 de 13 de outubro de 1969. Provê sobre as profissões de fisioterapeuta e terapeuta ocupacional e dá outras providências. Diário Oficial da União, Brasília, n.197, seção 1, p.3658. 16 out. 1969. Disponível em: http://www.camara.gov.br/sileg/integras/789483.pdf. Acesso em: 17 jan. 2017.

CATANI, Afrânio Mendes; OLIVEIRA, João Ferreira; DOURADO, Luiz Fernandes. Política educacional, mudanças no mundo do trabalho e reforma curricular nos cursos de graduação no Brasil. Educação \& Sociedade, Campinas, v.22, n.75, p.6783, 2001. Disponível em: http://dx.doi.org/10.1590/S010173302001000200006. Acesso em: 10 jan. 2017.

CECCIM, Ricardo Burg; CARVALHO, Yara Maria. Ensino da saúde como projeto da integralidade: a educação dos profissionais de saúde no SUS. In: PINHEIRO, R.; CECCIM, R. B., MATTOS, R. A. (Org.). Ensinar Saúde: a integralidade e o SUS nos cursos de graduação na área da saúde. 2.ed. Rio de Janeiro: IMS/UERJ: CEPESQ: ABRASCO, 2006. p.69-92.

CERVO. Amado Luiz; BERVIAN, Pedro Alcindo. Metodologia científica. São Paulo: Makron Books, 1996.

DA ROS, Marco Aurélio. Estilos de pensamento em saúde pública: um estudo da FSP-USP e ENSP-Fiocruz, entre 1948 e 1994, a partir da epistemologia de Ludwik Fleck. Florianópolis. 208fls. Tese (Doutorado em Educação) - Universidade Federal de Santa Catarina, 2000. Disponível em: http://repositorio.ufsc.br/xmlui/ handle/123456789/78913. Acesso em:12 jan.2017.

GONZÁLEZ, Alberto Durán; ALMEIDA, Marcio José de. Movimentos de mudança na formação em saúde: da medicina comunitária às diretrizes curriculares. Physis Revista de Saúde Coletiva, Rio de Janeiro, v.20, n.2, p.551-570, 2010. Disponível em: http://www.scielo.br/pdf/physis/v20n2/a12v20n2.pdf. Acesso em: 14 jan.2017.

LAKATOS, Eva Maria; MARCONI, Marina de Andrade. Metodologia científica. São Paulo: Atlas, 1987.

MARCO, Mario Alfredo de. Do Modelo Biomédico ao Modelo Biopsicossocial: Um Projeto de Educação Permanente. Revista Brasileira de Educação Médica. Rio de Janeiro, v.30, n.1, p. 60-67, 2006. Disponível em: http://dx.doi.org/10.1590/S0100-55022006000100010. Acesso em: 10 jan. 2017.

MEHRY, Emerson Elias; MALTA, Deborah Carvalho; SANTOS, Fausto Pereira dos. Desafios para os gestores do SUS hoje: compreender os modelos de assistência à saúde no âmbito da reforma sanitáriabrasileira e a potência transformadora da gestão. In: FREESE Eduardo. Municípios: a gestão da mudança em saúde. Recife: UFPE; 2004. p. 45-76.

MOREIRA, Carlos Otávio Fiuza; DIAS, Maria Socorro de Araújo. Diretrizes curriculares na saúde e as mudanças nos modelos de saúde e de educação. ABCS Health Science. v. 40, n.3, p.300-305, 2015. Disponível em: https:// www.portalnepas.org.br/abcshs/article/viewFile/811/706. Acesso em: 20 jan.2017.

PACHECO, José Augusto. Escritos Curriculares. São Paulo: Cortez, 2005. 176p.

PAGLIOSA, Fernando Luiz; DA ROS, Marco Aurélio. O relatório Flexner: para o bem e para o mal. Revista Brasileira de Educação Medica. vol.32, n.4, pp.492-499, 2008. Disponível em: http://dx.doi.org/10.1590/ S0100-55022008000400012. Acesso em 12 jan. 2017.

PAIM, Jairnilson Silva. Políticas de saúde no Brasil. In: ROUQUAYROL, Maria Zélia; ALMEIDA FILHO, Naomar (Org.). Epidemiologia \& Saúde. 6. ed. Rio de Janeiro: Medsi, 2003. p.587-603.

PAIM, Jairnilson Silva. Saúde: política e reforma sanitária. Salvador: ISC. 2002.

PARENTE, José Reginaldo Feijão. Preceptoria e tutoria na residência multiprofissional em saúde da família. Sanare.v.7, n.2, p.47-53, 2008. Disponível em: https://sanare.emnuvens.com.br/sanare/article/view/31. Acesso em: 20 jan. 2017. 
PEREIRA, Luciana Alves; ALMEIDA, Márcio. Fisioterapia. In: Fundação Oswaldo Cruz. Dinâmica das graduações em saúde no Brasil: subsídios para uma política de recursos humanos. Brasília: Ministério da Saúde. p.171-184, 2006.

PEREIRA, Ingrid D’avilla Freire. A formação profissional em saúde no Brasil pós-1988: aspectos das Conferências Nacionais de Saúde e das Políticas Públicas. 150 f. Dissertação (Mestrado em Saúde Pública). Escola Nacional de Saúde Pública Sergio Arouca, Rio de Janeiro, 2013.

PEREIRA, Thaís Thomé Seni Oliveira; BARROS, Monalisa Nascimento dos Santos; AUGUSTO, Maria Cecília Nobrega de Almeida. O Cuidado em Saúde: o Paradigma Biopsicossocial e a Subjetividade em Foco. Mental - ano IX - no 17 p. 523-536, Barbacena-MG - jul./dez. 2011. Disponível em: http://www.redalyc.org/articulo. oa?id=42023679002. Acesso em:20 jan. 2017.

REZENDE, Mônica de. Avaliação da Inserção do Fisioterapeuta na Saúde da Família de Macaé/RJ: A Contribuição deste Profissional para a Acessibilidade da População Idosa às Ações de Saúde da Equipe. Um Estudo de Caso. 145 f. Dissertação (Mestrado em Saúde Pública). Escola Nacional de Saúde Pública Sergio Arouca, Fundação Oswaldo Cruz, Rio de Janeiro, 2007. Disponpivel em: https://www.arca.fiocruz.br/handle/ icict/4638. Acesso em 10 jan. 2017.

RODRIGUES, Raquel Miguel. A fisioterapia no contexto da política de saúde no Brasil: aproximações e desafios. Perspectivas Online. v.2, n.8, 2008. Disponível em: http://www.seer.perspectivasonline.com.br/index.php/ revista_antiga/article/view/335/246. Acesso em:03 jan 2017.

ROSSONI, Eloá; LAMPERT, Jadete. Formação de profissionais para o Sistema Único de Saúde e as diretrizes curriculares. Boletim da Saúde. v.18, n.1, p.87-98, 2004. Disponível em: ttp://bvsms.saude.gov.br/bvs/ periodicos/boletim_saude_v18n1.pdf\#page=86. Acesso em: 03 jan. 2017.

SACRISTÁN, Gimeno. Saberes e incertezas sobre o currículo. Porto Alegre, Penso, 2013, 542p.

SAKAI, Márcia Hiromi. Recursos Humanos em Saúde. In: ANDRADE, S.M.; SOARES, D.A.; CORDONI JR., L. Bases da Saúde Coletiva. Londrina: EdUEL, 2001. p. 111-124.

SANTOMÉ, Jurgo Torres. A organização relevante dos conteúdos nos currículos. IN: Globalização e interdisciplinaridade: o currículo integrado. Porto Alegre: Ed. Artes Médicas Sul Ltda., 1998.

SANTOS, Adriana Regina de Jesus. Currículo e Educação: conceitos e questões no contexto educacional. In: CONGRESSO NACIONAL DE EDUCAÇÃO, n. 8, 2008. Curitiba - Paraná. Anais do VIII Congresso Internacional de Educação - EDUCERE Edição Internacional; Anais III Congresso Ibero Americano Sobre Violências nas Escolas - CIAVE. Curitiba: Champagnant, 2008. p. 2638-2651. Disponível em: http:// www.pucpr.br/eventos/educere/educere2009/anais/pdf/3384_1596.pdf. Acesso em: 17jan.2017.

SILVA, Jaqueline Regina Kaik; SILVA, Alaine Sousa. Fisioterapia no Município de Bragança Paulista: Caracterização do Serviço e do Perfil Epidemiológico dos Pacientes em Lista de Espera. Trabalho de Conclusão de Curso. 2010. Universidade São Francisco. Bragança Paulista, 2010.

SUMIYA, Alberto. A Fisioterapia como prática híbrida, as mudanças do currículo e o perfil profissional. 248fls. 2014. Tese (Doutorado em Ciências da Motricidade) - Universidade Estadual Paulista, Instituto de Biociências de Rio Claro, 2014. Disponível em: http://hdl.handle.net/11449/122130. Acesso em 22 jan. 2017.

UTIDA, Victor Hugo de Sousa; PAGANINI, Junyce; FAGUNDES, Rayne Ramos; AMARAL, Lívia Ellen França; OLIVEIRA, Valéria Rodrigues Costa. Estrutura Curricular dos Cursos de Fisioterapia do Estado de Goiás: Um destaque a saúde coletiva. Revista Movimenta. v. 5, n.4, 2012. 